\title{
Nattokinase crude extract enhances oral mucositis healing
}

\author{
Junyao Zhang, Yu Tang, Tao Yuan, Mengting Yang, Wenjing Fang, Li Li, Fei Fei and Aihua Gong*
}

\begin{abstract}
Background: Nattokinase (NK) is a promising alternative in the prevention and treatment of cardiovascular diseases due to its potent fibrinolytic activity. In this study, we investigated the effect of crude nattokinase extract on the healing of acetic acid-induced oral mucositis in mice.

Methods: Bacillus subtilis culture media (BSCM) was isolated into the supernatant, named nattokinase crude extract (NCE), and the pellet was named Bacillus subtilis mass (BSM). An oral mucositis model was established in mice by applying 50\% glacial acetic acid to the buccal mucosa. According to the treatment conditions, the mice were divided into BSCM, NCE, BSM and phosphate buffered saline (PBS) groups. The weight of the mice, oral mucositis healing score and histopathological examination were used to evaluate the treatment.

Results: Fibrinolytic activities of BSCM, NCE and BSM were approximately $8069,10,800$ and $80 \mathrm{U} / \mathrm{ml}$, respectively. The weight gain of mice in the NCE group was significantly different from the PBS group after three days' treatment $(p<0.05)$. The oral mucositis score of NCE group was significantly higher than other groups $(p<0.05)$. The differences in histopathology scores between the NCE and other groups were statistically significant $(p<0.01)$.

Conclusions: NCE could possess remarkable potential to reduce pain and promote oral mucositis healing with minimal safety concerns. In this study, we first report that NCE from the supernatant of Bacillus subtilis can promote the healing of oral mucositis, which extends the application scope of NK.
\end{abstract}

Keywords: Nattokinase, Bacillus subtilis, Mucosal epithelium, Oral mucositis healing

\section{Background}

Oral mucositis is one of the adverse effects associated with upper body radiotherapy or chemoradiotherapy $[1,2]$. During the initiation phase of mucositis, patients begin to develop erythema followed by focal areas of oral mucosal desquamation [3]. Then the progress of mucositis is prolonged and severe, the integrity of the mucosa is destroyed, and lesions begin to form [4]. These lesions can cause mild to severe pain, which often leads to swallowing disorders, result in weight loss in mice [5-7]. The pain is mainly stimulated by inflammatory mediators such as prostaglandins, 5-hydroxytryptamine and

*Correspondence: ahg5@ujs.edu.cn

Department of Cell Biology, School of Medicine, Jiangsu University, 301

Xuefu Road, Zhenjiang 212003, China bradykinin secreted by inflammatory cells [8]. At present, a combination of treatments, such as local rinses with $2 \%$ viscous lidocaine solution, mouthwash preparations and other systemic analgesics are used to control pain [9]. However, most commercially available mouthwashes contain alcohol and other ingredients, which sensitize the lesion and make patients uncomfortable. Therefore, there is a dire need to develop mild strategies to promote oral mucositis healing.

In 1987, Sumi et al. [10] first named a novel fibrinolytic enzyme NK, which was found in natto, a typical and popular fermented soybean food in a traditional Japanese diet. NK is the most important extracellular enzyme produced by Bacillus subtilis natto [11]. Both in vitro and in vivo studies have confirmed that NK has potent fibrinolytic activity [12]. Thus, NK is now considered an 
efficient, secure and economic enzyme that draws central attention in thrombolytic drug studies [13-16]. In addition, NK is also used in the treatment of Alzheimer's disease, vitreoretinal disorders and some tumours $[17,18]$. Clinical trials have also proven that increasing the intake of nattokinase is effective in preventing and treating hypertension [19].

Recent study revealed that NK exerts its anti-inflammatory activity by inhibiting LPS-induced activation of TLR4 and NOX2, which may help patients relieve the pain [20]. According to our previous research, NK enhances cell stemness and induces epithelial cell regeneration and angiogenesis, thereby promoting wound healing [21]. These results indicate that NK might be effective in oral mucositis healing.

In this study, we aimed to investigate the changes in body weight, inflammation and the progression of epithelial regeneration in mice to determine the role of NCE in the treatment of oral mucositis.

\section{Methods \\ Ethical approval}

All procedures performed in this study were in accordance with the relevant guidelines and regulations. The animal study was reviewed and approved by the Medical Ethics Committee and Ethics Committee for Experimental Animals of Jiangsu University (IRB protocol number: 2012258). Additionally, the authors followed all guidelines of The Animal Research: Reporting in vivo Experiments guidelines (ARRIVE).

\section{Medium and culture conditions}

The production of nattokinase mainly involves the following two steps: strain activation and fermentation. First, Bacillus subtilis natto was inoculated in seed culture medium (sodium chloride $2 \%$, peptone $1 \%$, yeast extract $0.5 \%$ and $100 \mathrm{~mL}$ of deionized water). The seed cultures were inoculated $(0.5 \%, \mathrm{v} / \mathrm{v})$ into a fermentation medium for NK production after cultivation in a thermostat at $37^{\circ} \mathrm{C}$ for $24 \mathrm{~h}$. The fermentation medium comprised $5 \%$ red bean powder, $2 \%$ glucose, $1 \%$ sodium chloride, $0.1 \%$ dipotassium hydrogen phosphate, $0.1 \%$ potassium dihydrogen phosphate, $0.01 \%$ calcium chloride and $0.05 \%$ magnesium sulphate. The fermentation media was contained in a conical flask and incubated in a shaker at $225 \mathrm{rpm}$ at $37^{\circ} \mathrm{C}$ for $72 \mathrm{~h}$.

\section{Isolation of NCE}

NCE was extracted from the fermentation medium, or BSCM. BSCM was centrifuged for $8 \mathrm{~min}$ at $12,000 \times \mathrm{g}$, the liquid supernatant was named NCE, and the pellet was resuspended in PBS and named BSM.

\section{Preparation of agarose-fibrin plate}

The fibrinolytic activity of NCE and other components was measured by the agarose-fibrin plate method. Briefly, the fibrin plate was made with a fibrinogen and agarose solution. Urokinase was used as the standard. The samples were dropped in the hole on the plate. The plate was incubated at $37^{\circ} \mathrm{C}$ for $18 \mathrm{~h}$. Finally, the fibrinolytic activity was determined from a standard curve.

\section{Mice oral mucositis model and treatment}

Adult male ICR mice (8 weeks old; weighing $25 \pm 2$ g) were purchased from the Animal Centre of Jiangsu University (Zhenjiang, China), housed in individual plastic cages and subjected to a 12-h light-dark cycle with $60 \%$ relative humidity at $25 \pm 2{ }^{\circ} \mathrm{C}$. A diameter of $2-\mathrm{mm}$ piece of filter paper was soaked in $50 \%$ acetic acid and placed in the labial fornix region of the inferior incisors of anaesthetized mice for $60 \mathrm{~s}$ to form the lesion [22, 23]. The mice were grouped into four groups, five mice one group. PBS treatment was used as a control group, and the other groups were treated with BSCM, NCE and BSM respectively. At 3, 6 and 10 days after treatment, we photographed oral mucositis lesions. Three calibrated and blinded observers were involved to evaluated the oral mucositis healing through specific evaluation criteria, and the interobserver variability was calculated. (0: model initial lesion, redness and grey pseudomembrane; 1: lesion destruction, unbleached pseudomembrane, obvious exudate, and redness; 2: lesion convergence, slight redness and less exudate; 3: significantly smaller lesion, a little exudate, a little redness; 4: completely healed lesion). Finally, the mice were sacrificed and the lesion tissue were resected for further analysis.

\section{Measurement of mice weight}

We recorded the weight of mice daily after treatment. The relative weight $=[(d n-d 0) / d 0](d 0$ is original weight of mice, and $d n$ is the weight of mice were recorded after $\mathrm{n}$ days).

\section{Histopathological examination}

The lesion tissue was fixed with $4 \%$ paraformaldehyde ( $\mathrm{pH}$ 7.4), gradually dehydrated and embedded in paraffin. Four-micrometer-thick sections were obtained with a microtome (Leica, RM2145, Germany) and stained with haematoxylin and eosin(H\&E) for assessing inflammatory response and histological changes. Images were captured with a microscope (Zeiss, Axio Scope A1, Germany). Three calibrated and blinded observers were involved to evaluated the histopathological images and the interobserver variability was calculated. H\&E stained lesions were analysed and scored through following 
evaluation criteria (0: vasodilatation, leukocyte infiltration and oral epithelial destruction; 0.5: vascular contraction, leukocyte decline and oral epithelial destruction; 1: vasoconstriction, leukocyte decline, oral epithelial destruction and a small amount of lymphocytes; 1.5 : vascular obviously destroyed, leukocyte not seen, oral epithelial destruction and a large number of lymphocytes; 2: normal vascular, leukocyte are not seen and a small number of lymphocytes; 2.5: normal vascular, leukocyte are not seen, healed oral epithelium and lymphocytes are not seen.).

\section{Statistical analysis}

Data are shown as the mean $\pm \mathrm{SD}$. Comparisons between groups were analysed using Student's t test (two groups) or one-way analysis of variance (ANOVA; multiple groups) using Prism 5.0 software (GraphPad, San Diego, CA). P values $<0.05$ were considered significant.

\section{Results}

\section{Detection of fibrinolytic activity}

The fibrinolytic activity of different components from Bacillus subtilis culture media was detected with agarose-fibrin plates. The results showed that the fibrinolytic activity of NCE was approximately $8069 \mathrm{U} / \mathrm{ml}$, the fibrinolytic activity of BSCM was approximately $10,800 \mathrm{U} / \mathrm{ml}$, and the fibrinolytic activity of BSM was only $80 \mathrm{U} / \mathrm{ml}$ (Fig. 1 ).

\section{NCE increases the relative weight of the oral mucositis mice}

An oral mucositis model was constructed in mice, and the relative weight of the mice after administration was used to evaluate the degree of oral mucositis healing. As shown in Fig. 2, the weight gain of mice in the NCE group was significantly different from the PBS group after three days' treatment $(p<0.05)$ The body weight of mice in other groups did not increase until the 5 th day after treatment.

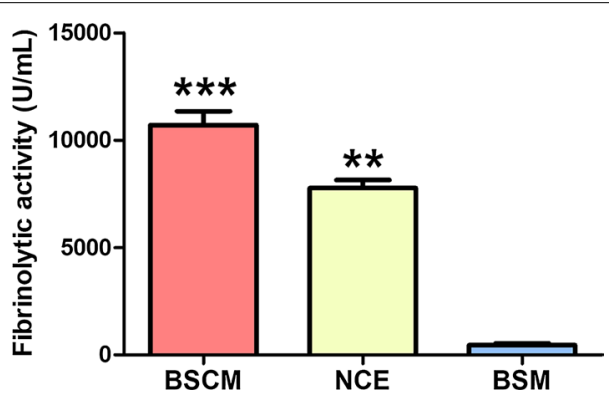

Fig. 1 Fibrinolytic activity detection with agarose-fibrin plates. $(n=3$, $\left.{ }^{* *} p<0.01,{ }^{* *} p<0.001\right)$

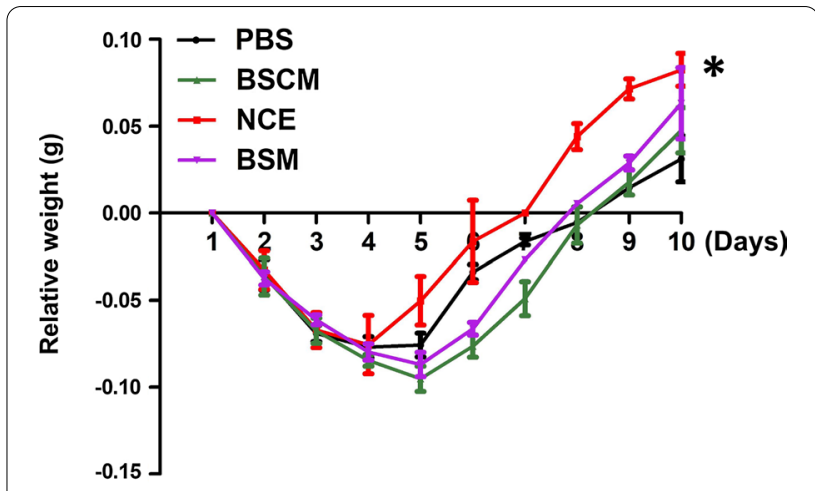

Fig. 2 NCE promotes the weight gain of the model mice. (NCE group compared with PBS group, ${ }^{*} p<0.05$ )

\section{NCE accelerates oral mucositis healing}

The general pictures showed that the area of oral mucositis in the NCE group was reduced and the exudate was less than that in the other three groups on the 3rd day after treatment. Surprisingly, the oral mucositis lesion in the NCE group healed compared with the other three groups on the 6th day after treatment. PBS did not promote oral mucositis healing (Fig. 3a). The score of oral mucositis was analysed as presented in Fig. 3b, the score of NCE group was significantly higher than the PBS group $(p<0.05)$. The comparison of oral mucositis scores is displayed in Table 1.

\section{NCE enhances mucosal epithelial regeneration in oral mucositis}

The results of $H \& E$ staining showed that the epithelial layer of each group was destroyed at $72 \mathrm{~h}$ after acetic acid treatment, accompanied by severe inflammatory cell infiltration. After 3 days of treatment, the NCE group had smaller lesions and reduced inflammatory cell infiltration compared with the other three groups. On the 6th day after the treatment, the epithelium in the NCE group recovered completely without inflammatory cell infiltration, while in the other three groups, the epithelium was not repaired, and there was still inflammatory cell infiltration (Fig. 4a). As depicted in Fig. 4b, the difference in histopathology score between the NCE group and the PBS group was statistically significant $(p<0.01)$.

\section{Discussion}

Here, we confirmed the role of NCE in oral mucositis healing. We found that NCE was closely correlated with body weight changes, inflammation control and epithelial regeneration in mouse oral mucositis model.

Clinical features of oral mucositis include diffuse mucosal erythema and ulcerations, accompanying with 


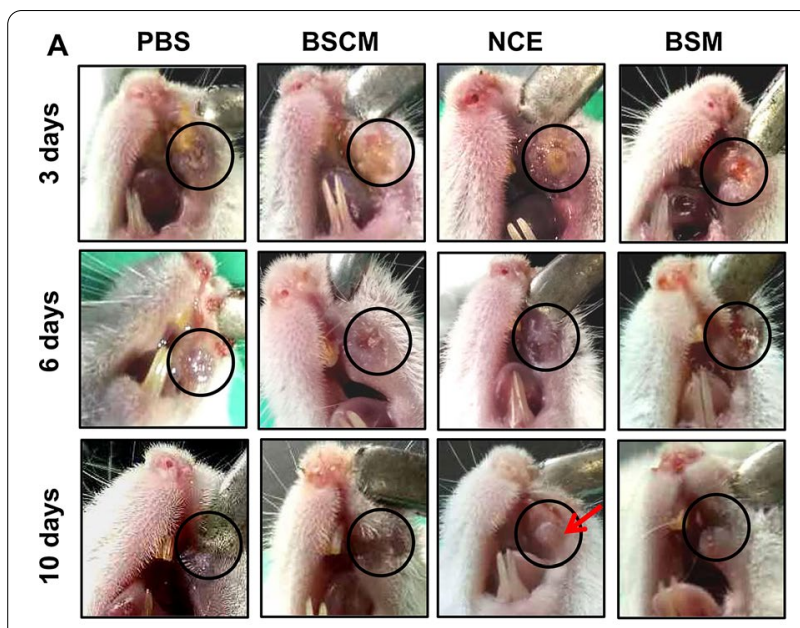

B

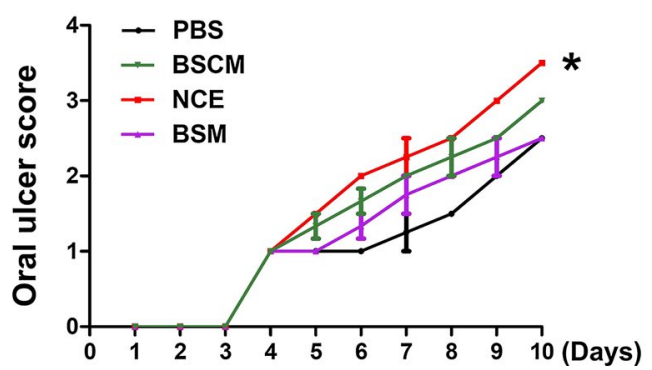

Fig. 3 NCE promotes oral mucositis healing. a Representative images of oral mucositis lesion on the 3rd, 6th and 10th days after treatment with PBS, BSCM, NCE and BSM. The circles mark the location of the lesion and the arrow indicates the healing of oral mucositis. $\mathbf{b}$ Oral mucositis scored through evaluation criteria $\left({ }^{*} p<0.05\right)$

mouth and throat pain [24], which interfere with eating and swallowing [25]. In particular, eating cold, hot, acidic and irritant foods often causes severe pain, which seriously affects quality of life [26]. Many interventions have been developed to treat oral mucositis, such as oral zinc sulphate, chlorhexidine, honey and laser therapy [27-29]. However, these interventions will bring additional pain to the patients. In this study, we found that the mouse weight of the NCE-treated group was increased significantly on the $4^{\text {th }}$ day after administration compared with that of the other groups, which indicated that NCE might relieve pain caused by oral mucositis and increase the mouse weight associated with eating and drinking.

Previous studies have confirmed that excessive inflammatory cells and factors participate in the formation of oral mucositis and stimulate sensory nerve endings [30]. Anti-inflammatory agents are regarded as the first-line agents to control the symptoms of oral mucositis. In our study, according to histological findings, the NCE group had better epithelial healing and moderate chronic inflammation on the 6th day than the other groups, which indicated that NCE can reduce excessive inflammatory responses and has a therapeutic effect on oral mucositis.

Speeding up the healing process, reducing pain and the chance of recurrence are the main aims of current therapy [31]. Several natural compounds can be administered as dietary supplements for the treatment of dermal and oral mucositis, our research extend this list [32]. As a food, NK has been widely proven to be safe, and toxicological assessments have also shown that the oral consumption of NK is of low toxicological concern [16].

Oral mucositis healing is a multifactorial process that involves inflammation, cell proliferation, migration and connective tissue remodelling [6]. Although we have demonstrated that NCE is effective in treating oral mucositis, the exact molecular mechanisms involved are still unknown and deserve further study.

\section{Conclusions}

NCE could possess remarkable potential to reduce pain and promote oral mucositis healing with minimal safety concerns. In this study, we first report that NCE from the supernatant of Bacillus subtilis can promote the healing of oral mucositis and should be a valid alternative to the conventional treatment.

Table 1 Comparison of oral mucositis scores between NCE group and PBS group

\begin{tabular}{|c|c|c|c|c|c|c|c|c|c|c|}
\hline \multirow[t]{2}{*}{ Group } & \multicolumn{10}{|l|}{ Time } \\
\hline & Day 1 & Day 2 & Day 3 & Day 4 & Day 5 & Day 6 & Day 7 & Day 8 & Day 9 & Day 10 \\
\hline PBS $(N=3)$ & - & - & - & 1 & 1 & 1 & 1.25 & 1.5 & 2 & 2.5 \\
\hline $\operatorname{NCE}(N=3)$ & - & - & - & 1 & 1.5 & 2 & 2.25 & 2.5 & 3 & 3.5 \\
\hline $\mathrm{BSM}(\mathrm{N}=3)$ & - & - & - & 1 & 1 & 1.3 & 1.75 & 2 & 2.25 & 2.5 \\
\hline $\operatorname{BSCM}(N=3)$ & - & - & - & 1 & 1.3 & 1.7 & 2 & 2.25 & 2.5 & 3 \\
\hline$P$ value & & & & & & $<0.05$ & & & & $<0.05$ \\
\hline
\end{tabular}

${ }^{\text {a }}$ Comparison between NCE and PBS 


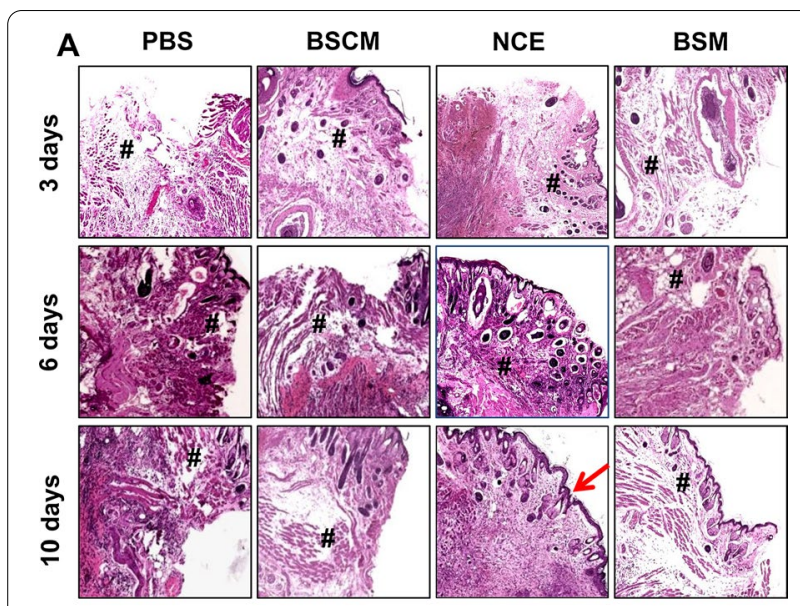

B

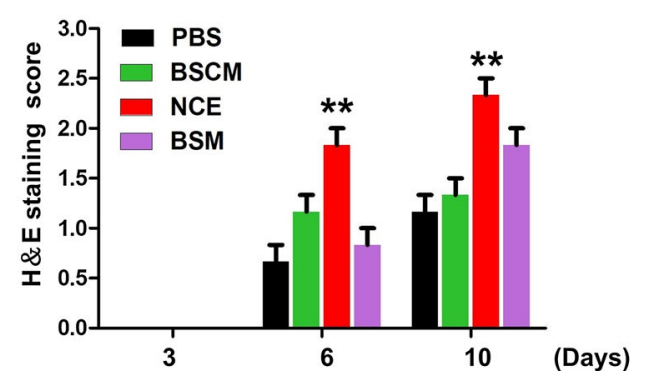

Fig. 4 NCE enhances re-epithelialization of oral mucositis. a Representative photomicrograph of H\&E staining of lesion tissue on the $3 \mathrm{rd}$, 6 th and 10 th days after treatment. The arrow indicates re-epithelialization, \# indicates inflammation. b Histopathological score of each group $\left.{ }^{* *} p<0.01\right)$

\section{Abbreviations}

BSCM: Bacillus subtilis culture media; BSM: Bacillus subtilis mass; H\&E: Haematoxylin and eosin; NCE: Nattokinase crude extract; NK: Nattokinase; PBS: Phosphate buffer saline.

\section{Acknowledgements}

Not applicable.

\section{Authors' contributions}

JZ and AG conceived the idea and designed the study. JZ and YT wrote the main manuscript text. TY prepared Figs. 1 and 2 and MY prepared Figs. 3 and 4. All listed authors participated in the experiments and data collection. All authors have read and approved the final manuscript.

\section{Funding}

This study was supported by the National Natural Science Foundation of China $(81772694,81372718)$.

\section{Availability of data and materials}

The various raw data and methods used to support the findings of this study are available from the corresponding author upon request.

\section{Declarations}

\section{Ethics approval and consent to participate}

All procedures performed in this study were in accordance with the relevant guidelines and regulations. The animal study was reviewed and approved by the Medical Ethics Committee and Ethics Committee for Experimental
Animals of Jiangsu University (IRB protocol number: 2012258). Additionally, the authors followed all guidelines of The Animal Research: Reporting in vivo Experiments guidelines (ARRIVE).

\section{Consent for publication}

Not applicable.

\section{Competing interests}

The authors declare no competing interests.

Received: 5 April 2021 Accepted: 10 October 2021

Published online:

\section{References}

1. Abdel Moneim AE, Guerra-Librero A, Florido J, Shen YQ, Fernandez-Gil B, Acuna-Castroviejo D, Escames G. Oral mucositis: melatonin gel an effective new treatment. Int J Mol Sci. 2017;18(5):1003.

2. Riley P, McCabe MG, Glenny AM. Oral cryotherapy for preventing oral mucositis in patients receiving cancer treatment. JAMA Oncol. 2016;2(10):1365-6.

3. Hong BY, Sobue T, Choquette L, Dupuy AK, Thompson A, Burleson JA, Salner AL, Schauer PK, Joshi P, Fox E, et al. Chemotherapy-induced oral mucositis is associated with detrimental bacterial dysbiosis. Microbiome. 2019;7(1):66

4. Onseng K, Johns NP, Khuayjarernpanishk T, Subongkot S, Priprem A, Hurst $C$, Johns J. Beneficial effects of adjuvant melatonin in minimizing oral mucositis complications in head and neck cancer patients receiving concurrent chemoradiation. J Altern Complem Med. 2017;23(12):957-63.

5. Fleckenstein J, Kuhne M, Seegmuller K, Derschang S, Melchior P, Graber S, Fricke $A$, Rube $C E$, Rube $C$. The impact of individual in vivo repair of DNA double-strand breaks on oral mucositis in adjuvant radiotherapy of headand-neck cancer. Int J Radiat Oncol Biol Phys. 2011;81(5):1465-72.

6. Nodai T, Hitomi S, Ono K, Masaki C, Harano N, Morii A, Sago-Ito M, Ujihara I, Hibino T, Terawaki K, et al. Endothelin-1 elicits TRP-mediated pain in an acid-induced oral ulcer model. J Dent Res. 2018;97(8):901-8.

7. Khanjani Pour-Fard-Pachekenari A, Rahmani A, Ghahramanian A, Asghari Jafarabadi M, Onyeka TC, Davoodi A. The effect of an oral care protocol and honey mouthwash on mucositis in acute myeloid leukemia patients undergoing chemotherapy: a single-blind clinical trial. Clin Oral Investig. 2019;23(4):1811-21.

8. Ronchetti S, Migliorati G, Delfino DV. Association of inflammatory mediators with pain perception. Biomed Pharmacother. 2017:96:1445-52.

9. Descroix V, Coudert AE. A viscous lidocaine mouthwash is no better than placebo for improving oral intake in children with painful mouth ulcers. Evid Based Med. 2014;19(5):179.

10. Sumi $H$, Hamada $H$, Tsushima $H$, Mihara H, Muraki H. A novel fibrinolytic enzyme (nattokinase) in the vegetable cheese Natto; a typical and popular soybean food in the Japanese diet. Experientia. 1987;43(10):1110-1.

11. Dabbagh F, Negahdaripour M, Berenjian A, Behfar A, Mohammadi F, Zamani M, Irajie C, Ghasemi Y. Nattokinase: production and application. Appl Microbiol Biotechnol. 2014;98(22):9199-206.

12. Cho YH, Song JY, Kim KM, Kim MK, Lee IY, Kim SB, Kim HS, Han NS, Lee BH, Kim BS. Production of nattokinase by batch and fed-batch culture of Bacillus subtilis. New Biotechnol. 2010;27(4):341-6.

13. Wu C, Gao C, Lu S, Xu X, Wen N, Zhang S, Liu M. Construction of polylysine dendrimer nanocomposites carrying nattokinase and their application in thrombolysis. J Biomed Mater Res A. 2018;106(2):440-9.

14. Huang Y, Ding S, Liu M, Gao C, Yang J, Zhang X, Ding B. Ultra-small and anionic starch nanospheres: formation and vitro thrombolytic behavior study. Carbohydr Polym. 2013;96(2):426-34.

15. Berenjian A, Mahanama R, Talbot A, Regtop H, Kavanagh J, Dehghani F. Designing of an intensification process for biosynthesis and recovery of menaquinone-7. Appl Biochem Biotechnol. 2014;172(3):1347-57.

16. Lampe BJ, English JC. Toxicological assessment of nattokinase derived from Bacillus subtilis var. natto. Food Chem Toxicol. 2016;88:87-99.

17. Kou Y, Feng R, Chen J, Duan L, Wang S, Hu Y, Zhang N, Wang T, Deng Y, Song Y. Development of a nattokinase-polysialic acid complex for advanced tumor treatment. Eur J Pharm Sci. 2020;145:105241. 
18. Yan Y, Wang Y, Qian J, Wu S, Ji Y, Liu Y, Zeng J, Gong A. Nattokinase crude extract inhibits hepatocellular carcinoma growth in mice. J Microbiol Biotechnol. 2019;29(8):1281-7.

19. Bhatt PC, Verma A, Al-Abbasi FA, Anwar F, Kumar V, Panda BP. Development of surface-engineered PLGA nanoparticulate-delivery system of Tet1-conjugated nattokinase enzyme for inhibition of Abeta40 plaques in Alzheimer's disease. Int J Nanomed. 2017;12:8749-68.

20. Wu H, Wang Y, Zhang Y, Xu F, Chen J, Duan L, Zhang T, Wang J, Zhang F. Breaking the vicious loop between inflammation, oxidative stress and coagulation, a novel anti-thrombus insight of nattokinase by inhibiting LPS-induced inflammation and oxidative stress. Redox Biol. 2020;32:101500

21. Zhang B, Liu Y, Ji Q, Zhao M, Zeng J, Liu L, Xu X, Yan Y, Gong A. Nattokinase crude extract enhances cutaneous wound healing. J Biomater Tissue Eng. 2017;7(12):1281-6.

22. Ossama M, Lamie C, Tarek M, Wagdy HA, Attia DA, Elmazar MM. Management of recurrent aphthous ulcers exploiting polymer-based Muco-adhesive sponges: in-vitro and in-vivo evaluation. Drug Deliv. 2021;28(1):87-99.

23. Karavana Hizarcioglu SY, Sezer B, Guneri P, Veral A, Boyacioglu H, Ertan G, Epstein JB. Efficacy of topical benzydamine hydrochloride gel on oral mucosal ulcers: an in vivo animal study. Int J Oral Maxillofac Surg. 2011;40(9):973-8.

24. Treister N, Nieder M, Baggott C, Olson E, Chen L, Dang H, Krailo M, August A, Sung L. Caphosol for prevention of oral mucositis in pediatric myeloablative haematopoietic cell transplantation. Br J Cancer. 2017;116(1):21-7.

25. Shen Z, Wang J, Huang Q, Shi Y, Wei Z, Zhang X, Qiu Y, Zhang M, Wang $Y$, Qin W, et al. Genetic modification to induce CXCR2 overexpression in mesenchymal stem cells enhances treatment benefits in radiationinduced oral mucositis. Cell Death Dis. 2018;9(2):229.

26. Saito H, Watanabe $Y$, Sato K, Ikawa H, Yoshida Y, Katakura A, Takayama $\mathrm{S}$, Sato M. Effects of professional oral health care on reducing the risk of chemotherapy-induced oral mucositis. Support Care Cancer. 2014;22(11):2935-40.

27. Munstedt $\mathrm{K}$, Mannle $\mathrm{H}$. Using bee products for the prevention and treatment of oral mucositis induced by cancer treatment. Molecules. 2019;24(17):3023.

28. Bayer S, Kazancioglu HO, Acar AH, Demirtas N, Kandas NO. Comparison of laser and ozone treatments on oral mucositis in an experimental model. Lasers Med Sci. 2017:32(3):673-7.

29. Alinca SB, Saglam E, Kandas NO, Okcu O, Yilmaz N, Goncu B, Dogan MA. Comparison of the efficacy of low-level laser therapy and photodynamic therapy on oral mucositis in rats. Lasers Med Sci. 2019;34(7):1483-91.

30. Boisvert WA. The participation of inflammatory cells in atherosclerosis. Drugs Today (Barc). 2001;37(3):173-9.

31. Viet CT, Corby PM, Akinwande A, Schmidt BL. Review of preclinical studies on treatment of mucositis and associated pain. J Dent Res. 2014:93(9):868-75.

32. Pulito C, Cristaudo A, Porta C, Zapperi S, Blandino G, Morrone A, Strano S. Oral mucositis: the hidden side of cancer therapy. J Exp Clin Cancer Res. 2020;39(1):210

\section{Publisher's Note}

Springer Nature remains neutral with regard to jurisdictional claims in published maps and institutional affiliations.
Ready to submit your research? Choose BMC and benefit from:

- fast, convenient online submission

- thorough peer review by experienced researchers in your field

- rapid publication on acceptance

- support for research data, including large and complex data types

- gold Open Access which fosters wider collaboration and increased citations

- maximum visibility for your research: over $100 \mathrm{M}$ website views per year

At BMC, research is always in progress.

Learn more biomedcentral.com/submissions 